[Comentarios al libro «Competitividad: Teoría y Politica», de Diego Gurrero]

Ariel - Barcelona, 1995

\title{
UNA NUEVA TEORIA DE LA COMPETENCIA REGIONAL, NACIONAL E INTERNACIONAL
}

EMILIO DIAZ CATLEJA

Este libro del profesor Diego Guerrero Competitividad: teoria y polttica constituye un excelente marco de reflexión sobre la naturaleza de la competencia en las sociedades occidentales contemporáneas, cuyo objetivo último es propiciar un debate abierto sobre la capacidad explicativa de la teoría de la competencia dominante, y por tanto sobre el grado de adecuación de las políticas macro y microeconómicas de competitividad, para sentar las bases de un análisis económico de la competencia y competitividad alternativo, directamente inspirado en la concepción de la escuela clásica de Economía Política, que el autor propone reformular rigurosamente. Guerrero parte de la necesidad de superar la concepción convencional de la competencia, fuertemente vinculada al principio ricardiano de la ventaja comparativa de costes, necesidad derivada de sus inconsistencias lógicas y empíricas, en la búsqueda de una alternativa teórica completa, que el autor identifica con el principio de la ventaja absoluta de costes. En este sentido, aunque buena parte del libro se dedica a análisis de la competencia y del comercio internacional, los planteamientos básicos de esta teoría de la competencia pueden ser aplicados fácilmente al ámbito nacional y regional, ya que el punto de partida del análisis es la primacia de los factores reales, frente a los factores monetarios, en la determinación de la capacidad de competir en el ámbito de la economía de mercado, y por tanto en la determinación del desarrollo desigual tanto a escala internacional como a escala nacional, la problemática que nás nos interesa en este contexto, y a la que por tanto dedicaremos mayor atención.

En este sentido, si se quieren entender los desequilibrios comerciales en el contexto del desarrollo desigual a escala nacional y mundial, es obligado no rechazar la posibilidad de que estos fenómenos sean precisamente consecuencia del libre comercio. Si se sigue este razonamiento, se ponen de inmediato de manifiesto las profundas limitaciones teóricas en el interior del análisis económico tradicional, y no sólo en lo que toca al argumento utilizado por Ricardo en su formulación del principio de la ventaja comparativa de costes, sino a todos sus sutitutos modernos en el ámbito de la competencia a escala mundial, pues «simplificando al máximo... podría afirmarse, por una parte, que añadiendo a 
los tres componentes ricardianos conocidos - a saber: ventaja comparativa, rechazo de la teoría del valor-trabajo y automatismo monetario- la teoría de la competencia perfecta lo que tenemos es el modelo neoclásico predominante, más conocido como modelo de Heckscher-Ohlin. Y si a los citados tres elementos se le añade la teoría de la competencia imperfecta, en vez de competencia perfecta, a lo que arribaremos será a alguna versión de las llamadas 'nuevas teorías del comercio internacional' (NTCI)" (pág. 39; énfasis añadido).

Las inconsistencias lógicas fundamentales apuntan, como se ha dicho, a la teoría básica de la competencia en el enfoque convencional moderno, siendo que las limitaciones teóricas no sólo se refieren a la competencia perfecta sino también y por idénticas razones, a la competencia imperfecta. Para mostrarlo, Guerrero desbroza pacientemente el núcleo axiomático del modelo de competencia convencional, poniendo de manifiesto las limitaciones de la estática comparativa, con la que no es posible dar entrada al tiempo real, ya que la dinámica exige introducir el cambio técnico como elemento endógeno en la organización económica, lo cual no es en último término compatible con la teoría de valor-utilidad en que se basa el modelo ni, por tanto, con la «tesis de la simetría» implícita en la ley de la oferta y la demanda. Por otra parte, la empresa capitalista es caracterizada bien como un mecanismo completamente pasivo que acepta costes y precios (competencia perfecta), o bien parcialmente pasivo, al aceptar costes pero determinar en mayor o menor grado los precios (competencia monopolística); en todo caso, no se plantea la posibilidad de empresas coste-determinantes, que es precisamente lo que está en la base de la posibilidad de competir en el sentido propio, es decir, tratar de incrementar la cuota de mercado y las condiciones de rentabilidad de la empresa abaratando el precio individual con respecto a un precio de mercado generalmente dado a corto plazo, cosa que depende esencialmente del incremento de productividad asociado al cambio técnico (mecanización), cuya centralidad en el análisis permitiría concebir teóricamente la competencia en proceso dinámico de agresivo enfrentamiento de las empresas, donde no es posible que todos puedan ganar ni por tanto un equilibrio permanente, como sucede en la realidad.

El autor desarrolla simultáneamente a este análisis lo que denomina "competencia dinámica intrasectorial", que pone el énfasis, frente a los modelos tradicionales, en la diversidad de técnicad dentro de las ramas que producen un mismo producto, lo cual origina diversidad en las estructuras de coste de las empresas $\mathrm{y}$, por tanto también diversidad en las tasas de rentabilidad entre las empresas del mismo sector (pág. 73). Para Guerrero, el modelo de competencia neoclásico, que pone el énfasis en la competencia intersectorial, es incompatible con la dispersión de las tasas de rentabilidad de las empresas dentro de cada sector, y rechaza categóricamente que los 
precios puedan ser determinados a largo plazo única y exclusivamente por los costes de producción unitarios (en cuyo caso, la demanda sólo determina la cantidad de producto intercambiada, pero no el precio), debido a la tesis de la simetría, cuya defensa descansa en la noción de economías (deseconomías) de escala y en la hipótesis de costes crecientes para cada escala de producción como caso general. Para Guerrero, es precisamente este marco conceptual artificial el que, al ligar los costes al tamaño de la empresa, pero manteniendo una técnica de producción única (utilizada a distinta «escala) para todas las empresas dentro de cada sector, garantizando así el equilibrio general sin invalidar el equilibrio parcial, invalida las leyes sobre los rendimientos, que dejan así de depender del progreso económico en general y del cambio técnico en particular, como sostenían los clásicos (págs. 63-66). Sin embargo, como muestra el autor, incluso respetando la lógica del modelo neoclásico (tanto de competencia perfecta como imperfecta), estas conclusiones no se sostienen cuando se introduce (aunque sea ex post) el cambio técnico (véase, en particular, págs. 67-72), siendo por otra parte este último endógenamente consustancial a la organización económica de nuestra sociedad.

Este análisis concluye con una primera aproximación a la teoría de la competencia dinámica (lo que para el autor constituye un "retorno a la concepción clásica de la competencia", de ahí que pueda hablarse de un "enfoque de economía política", frente a los enfoques convencionales), donde se admite la diversidad de técnicas de producción dentro de cada sector, mostrando cómo se forman las distintas categorías de precios ("precios directos», «precios de producción" y "precios de intervención») tanto en el marco intra-industrial como en el inter-industrial, basándose en las nociones dinámicas de «centros de gravedad" y «transferencia de excedentes» entre empresas y sectores (véase el epígrafe 2.5, págs. 72-77). Si bien no se trata de un modelo completamente dinámico, porque no se introduce el cambio técnico, sí perfila las condiciones de la competencia dinámica: la primacía de las condiciones de producción en la determinación de los precios en condiciones de libre movilidad del capital, la dispersión de la rentabilidad y de las estructuras de costes intra-industriales, la posibilidad de ganancias y pérdidas de cuotas de mercado en la competencia inter-industrial sobre la base de la formación de una tasa de ganancia media y las subsiguientes transferencias de excedente, los efectos derivados de las "restricciones de entrada", etc...

El presupuesto básico del que parte el «enfoque de economía política» es que «la competencia en el plano internacional se rige por las mismas leyes y principios que en el ámbito (nacional o) general" (pág. 117), de manera que la confluencia de distintas monedas en este ámbito complica, pero no altera, la naturaleza de los procesos competitivos. Es lícito, pues, partir del modelo de competencia dinámica en el marco de un área competitiva con una 
sola moneda para, posteriormente, introducir la mayor complejidad derivada de la existencia en un área competitiva de dos o más monedas. Este es precisamente el procedimiento seguido por el profesor Guerrero, que sigue aquí expresamente los planteamientos del economista norteamericano Awnar Shaikh, pionero en el desarrollo de la teoría del comercio internacional basada en las ventajas absolutas de coste. El análisis empieza, pues, con el modelo shaikhiano de determinación de los precios de producción en condiciones de competencia dinámica intra e interindustrial, que es una extensión dinámica de la aproximación presentada en el capítulo segundo, pero introduciendo dos ideas adicionales, estrechamente unidas: (i) la preeminencia de los capitales reguladores en la fijación de los "centros de gravedad" de los precios de producción, y (ii) la integración vertical de los coeficientes técnicos y económicos de producción.

Aplicado al "caso internacional", el modelo predice que "salvo en el caso de que las tasas de inflación sean muy altas, o que se trate de paises muy similares, no hay ninguna razón para esperar que los tipos de cambio se muevan conforme prevé la teoría de la paridad del poder adquisitivo, ni razón alguna por tanto para que el determinante a largo plazo del tipo de cambio real de dos monedas lleve a éste a tender hacia un valor igual a la unidad", y ello "ni siquiera cuando el tipo de cambio real de que se trate sea el referido a los bienes comerciables exclusivamente" (pág. 121), argumento este último que constituye la piedra angular con que el enfoque convencional pretende salvar el principio de la paridad del poder adquisitivo. La clave de esta conclusión radica en que el tipo de cambio real entre dos monedas en un área de competencia depende tendencialmente de la razón los costes laborales reales unitarios verticalmente integrados de cada país; es decir, el país donde el nivel de estos costes sea inferior tendrá ventaja absoluta con respecto al país competidor, de manera que el tipo de cambio real de su moneda será inferior a la unidad, cosa que le permitirá ganar cuotas de mercado (lo contrario ocurrirá para el país competidor, con capitales reguladores menos eficientes); las diferencias en lo que se refiere al nivel relativo de precios, para la economía en su conjunto y para los sectores comerciables y no comerciables, pueden matizar pero en absoluto invalidar la pauta tendencial del tipo de cambio real.

A partir de este modelo, el autor desarrolla una aproximación empíricamente operativa de los determinantes del tipo de cambio real y de la competitividad internacional introduciendo costes laborales unitarios convencionales y el ratio entre el índice de precios de consumo (que afecta al valor de los salarios reales) y el índice de precios de la totalidad del producto nacional (deflactor del PIB). De este análisis resulta que el tipo de cambio real entre dos monedas depende a largo plazo de la "cuota salarial relativa", esto es, del cociente entre las proporciones de la masa salarial y el PIB en cada país. Es 
decir; er*ij $*^{*}=\left(W_{i} / Y_{i}\right) /\left(W_{j} / Y_{j}\right)$, donde er ${ }^{*}$ ij representa el tipo de cambio real de la moneda del páis «i» con respecto a la moneda del país «j", “W" representa la masa salarial e "Y" el producto nacional (PIB). De esta forma: "puede afirmarse con todo rigor que el nivel medio de ventaja absoluta de un país (comparado ya sea con otro país individual, ya sea con cualquier conjunto de países, pertenezca o no el primero a dicho conjunto) viene sencillamente representado por el nivel comparativo que representan sus cuotas de salarios en el PIB» (págs. 126-127).

Pero obsérvese que en el caso «nacional» (tomando las regiones como áreas de competencia en el ámbito de una moneda única), donde los tipos de cambio no juegan por tanto papel alguno, se sigue manteniendo el principio básico de la ventaja competitiva de costes absolutos, lo que está lógicamente detrás de la posibilidad de desarrollo desigual a escala nacional y de la generación de patrones de intercambio estructuralmente desequilibrados. De esta forma, como a escala nacional el tipo de cambio no varía, pues se trata de un área de moneda única, abstrayéndonos de los diferenciales regionales de inflación, la capacidad de competir dependerá del nivel de los costes laborales unitarios relativos, que, en términos nominales, vienen dados por (págs. 122 y ss.):

$$
\mathrm{CLNU}_{\mathrm{ij}}=\mathrm{CLNU} / \mathrm{CLNU}_{\mathrm{j}}=\left\{\left(\mathrm{W}_{\mathrm{i}} / \mathrm{Y}_{\mathrm{i}}\right) *\left(\mathrm{Ot} \mathrm{t} / \mathrm{Oa}_{\mathrm{i}}\right) * \mathrm{dY} \mathrm{Y}_{\mathrm{i}}\right\} /\left(\left(\mathrm{W}_{\mathrm{j}} / \mathrm{Y}_{\mathrm{j}}\right) *\left(\mathrm{Ot} \mathrm{t} / \mathrm{Oa}_{\mathrm{j}}\right)^{*} \mathrm{dY}_{\mathrm{j}}\right\}
$$

Donde CLNU $\mathrm{ij}_{\mathrm{ij}}$ representa la ratio de los costes laborales unitarios nominales de la región «i" (CLNUi) sobre los costes laborales unitarios nominales de la región "j» (CLNUj), que es igual al nivel comparativo de las cuotas de salarios en el PIB ajustadas por las respectivas cuotas de ocupados totales (Ot) entre ocupados asalariados $(\mathrm{Oa})$, razón inversa de la tasa de ocupación salarial, y dY el deflactor del PIB de cada región. Mas los costes laborales unitarios nominales relativos son en realidad determinados por los costes laborales unitarios reales relativos $\left(C L R U_{i j}=C L R U_{i} / C L R U_{j}\right)$ y, a corto plazo, los niveles relativos de precios al consumo (ipcj/ipcj):

$$
\mathrm{CLNU}_{\mathrm{ij}}=\left(\dot{C L R U}_{\mathrm{i}} / \mathrm{CLRU}_{\mathrm{j}}\right)^{*}\left(\mathrm{ipc}_{\mathrm{i}} / \mathrm{ip \textrm {p } _ { \mathrm { j } }}\right)
$$

Finalmente, la evidencia empírica suministrada por el autor, tanto para la industria manufacturera como para la economía en su conjunto (en este último caso incluyendo la economía española) corrobora a grandes rasgos los planteamientos teóricos sostenidos en este libro. Con todo, debe añadirse que las limitaciones actuales en el terreno de la investigación aplicada en este ámbito no permiten ofrecer pruebas empíricas irrefutables. Lo que no cabe duda ninguna es que, a partir de la nueva teoría de la competencia dinámica 
las grandes anomalías a las que se enfrenta la teoría de la competencia tradicional (en sus diversas vertientes) son correctamente explicadas sin recurrir al dudoso expediente de negar la preeminencia de costes y precios en la lucha competitiva. El caso de Japón es, en nuestro criterio, ilustrativo a este respecto, pues el que este país "haya experimentado las más altas ganancias de cuotas del mercado mundial en el último medio siglo al mismo tiempo que elevaba más rápidamente que ningún país sus salarios nominales y reales y sus costes laborales unitarios relativos no es por tanto ninguna casualidad, sino un resultado que refleja fielmente las previsiones de la teoría expuesta aquín (pág. 195). 